\title{
Seroprevalence of Lawsonia intracellularis antibodies in intensive pig farms in China
}

\author{
Zongxue Wu¹, Yong Ling ${ }^{1}$, Deyu Tian', Qing Pan', Peter M H Heegaard² and Cheng $\mathrm{He}^{1 *}$
}

\begin{abstract}
Background: Porcine proliferative enteropathy caused by Lawsonia intracellularis (L. intracellularis) is a major concern to the pig industry worldwide. Although 8.3 billion pigs are produced each year in China, few reports on the prevalence of L.intracellularis infection are available. The aim of the current study was to estimate the seroprevalence of $L$. intracellularis antibodies in intensive pig farms in China.

Results: A total of 1060 serum samples were collected from 14 commercial pig farms located throughout China. Animals from all age groups were sampled including pre-weaning piglets, weaners, fattening pigs, adult sows and boars. Antibodies against L. intracellularis were detected using a specific blocking ELISA. Of the 1060 serum samples, 602 were identified as positive using the ELISA test. The apparent seroprevalence of L. intracellularis seropositivity was $57 \%$ (95\% Cl 50 to 64\%). The true prevalence (that is, prevalence corrected for the imperfect sensitivity and specificity of the testing method) was $77 \%$ (95\% Cl 70 to $83 \%$ ).

Conclusions: The highest true prevalence was observed in sows and boars, suggesting that within a herd these stock classes are a reservoir for infection. The prevalence of $L$. intracellularis seropositivity in local breed pigs was significantly less than that in imported breeds. A higher seroprevalence was found in pigs in herds in Central and Northern China, which may correspond to the greater use of the intensive production systems in these areas. We conclude that $L$. intracellularis is widely prevalent in commercial pigs in China.
\end{abstract}

Keywords: Lawsonia intracellularis, Prevalence, Antibody, Pigs, China

\section{Background}

Lawsonia intracellularis (L. intracellularis) is a gramnegative, fastidious obligate intracellular bacterium. The pathogen mostly occupies the small and large intestine in pigs and causes porcine proliferative enteropathy (PPE) post weaning. Infection leads to diarrhea, retarded growth and/or sudden death in fattening pigs. A high prevalence of $L$. intracellularis has been reported throughout the world, contributing to a substantial level of economic loss in the swine industry [1-3]. In some herds, the disease may manifest itself as severe hemorrhagic diarrhea with relative high mortality [4].

The first case of $L$. intracellularis infection in pigs was described in 1931 [5] and since that time, L. intracellularis has been reported in swine producing countries all over

\footnotetext{
* Correspondence: hecheng@cau.edu.cn

'Key Lab of Animal Epidemiology and Zoonosis of Ministry of Agriculture, College of Veterinary Medicine, China Agricultural University, Beijing 100193 , China

Full list of author information is available at the end of the article
}

the world. In Denmark, 94\% of tested animals were positive by PCR on feces [6], in Sweden $48 \%$ of herds were positive in fecal samples tested using nested PCR [7] and in Korea [8] 47\% of herds were positive when fecal samples were tested using multiplex PCR. More recent reports indicated a $100 \%$ seropositivity in Korea [9], $91 \%$ in the USA [10] and $84 \%$ in Australia [11]. In Australia $L$. intracellularis has been estimated to cost the industry in the order of USD 25 per sow annually [12] and direct losses of USD 3 to 11 per affected animal [13].

Highly intensive management of domestic pigs is widely promoted in mainland China, where there is an estimated pig population of approximately 8.3 billion [14]. Severe diarrhea occurs frequently in fattening pigs and pregnant sows, having negative impacts on herd feed conversion rates and herd profitability. Importantly, L. intracellularis infection receives comparatively little attention from animal health authorities compared to highly pathogenic infections such as porcine reproductive and respiratory
C Biomed Central

(c) 2014 Wu et al.; licensee BioMed Central Ltd. This is an Open Access article distributed under the terms of the Creative Commons Attribution License (http://creativecommons.org/licenses/by/2.0), which permits unrestricted use, distribution, and reproduction in any medium, provided the original work is properly credited. 
syndrome (PRRS), classical swine fever (CSF) and Streptococcus suis infection.

In 2008, the first isolate of $L$. intracellularis was identified from the intestinal mucosa of infected pigs in Southern China [15]. The apparent prevalence of L. intracellularis infected pigs identified by a PCR method was $14 \%$ and $16 \%$, in weaners and finishers, respectively in Guangxi province, Southern China [15]. To the best of the authors' knowledge the prevalence of $L$. intracellularis infection in pigs in other areas of China has not been reported. Moreover, the major transmission routes are unclear in different stock classes and production systems. In this study our aim was to determine the seroprevalence of L. intracellularis in pigs raised in the major pig-producing provinces in China. An additional aim of our study was to document the association between $L$. intracellularis positivity and the presence of diarrhea.

\section{Methods}

A cross-sectional survey carried out between January and May 2011 to estimate the seroprevalence of $L$. intracellularis infection in Chinese pigs. Seven provinces (Beijing, Hebei, Tianjin, Henan, Hubei, Guangdong and Guanxi) took part in the study. Sampling was carried out using a two-stage cluster design. Two herds from each of the seven provinces were selected at random from a sampling frame of 55 intensive pig cooperatives listed by the Chinese Ministry of Agriculture. Sample size calculations were carried out to determine the appropriate number of individual pigs to be sampled from each of the selected herds. Previous reports have estimated the within-herd prevalence of $L$. intracellulari infection to be around 13\% in weaning pigs and $16 \%$ in finishing pigs [15]. Based on a previous report [16], a credible estimate of the intra-cluster correlation coefficient for L. intracellularis infection was 0.06. We assumed an average cluster (i.e. herd) size of 80 pigs. Sample size calculations were carried out on the basis that we wanted to be $95 \%$ certain that our final estimate of $L$. intracellularis prevalence was within $5 \%$ of the true population value.

A total of 1060 serum samples were collected from pigs from the 14 herds that took part in the study. This included 147 sera from pre-weaning piglets ( 2 to 4 weeks of age), 221 sera from weaned piglets ( 5 to 7 weeks), 279 sera from fattening pigs ( 8 to 14 weeks), 255 sera from adult sows, and 158 sera from boars. Landrace and Large White (breeds exotic to China) and domestic breeds (Meishan and Jinhua) were represented in the sample. The pig numbers were 332, 287, 211 and 230 for Landrace, Large White, Meishan and Jinhua pigs, respectively. Serum samples were stored at $-80^{\circ} \mathrm{C}$ until assayed.

To identify the association between $L$. intracellularis seropositivity and the presence of diarrhea, 659 sera were obtained from herds with animals having a recent history of clinical diarrhea while an additional 401 samples were collected from herds without a recent history of diarrhea.

All sera were tested for antibodies against $L$. intracellularis using a blocking ELISA purchased from Synbiotic Europe SAS (Lyon, France) according to the manufacturer's instructions. The sensitivity and specificity of this test was $72 \%$ and $93 \%$, respectively [17].

The apparent prevalence of $L$. intracellularis seropositivity was calculated using the number of pigs identified as seropositive divided by the total number of pigs tested. Apparent prevalence estimates were then converted to true prevalence estimates using the approach proposed by Rogan and Gladen [18]. The Rogan and Gladen approach was used in this situation (in preference to more complex Bayesian methods) because the apparent prevalence estimates were relatively high. Confidence limits for the apparent and true prevalence estimates were determined using the Wilson binomial approximation [19,20]. Confidence intervals for both the apparent and true prevalence estimates were adjusted using the design effect, a function of the intra-cluster correlation coefficient for $L$. intracellularis infection and the average cluster size. The significance of differences between the true prevalence $L$. intracellularis seropositivity for pigs in different groups (i.e. location, stock class and breed) was assessed by considering their confidence limits. If the confidence intervals for two groups did not overlap, the inference was that the two prevalence estimates were significantly different at the alpha level of 0.05 [21]. All the calculations were performed using the AusVet epiTools procedures [22].

This study was approved by the Ethical Committee for Animal Experiments at China Agricultural University, Beijing, China.

\section{Results}

L. intracellularis antibodies were found in pigs of all ages. The true prevalence of $L$. intracellularis seropositivity was $77 \%$ (95\% CI 70 to 83\%). The true prevalence of $L$. intracellularis seropositivity ranged from $68-87 \%$ on stock class.

With respect to provinces, true prevalence of seropositivity varied, ranging from $43 \%$ (95\% CI 22 to $64 \%$ ) in Guanxi, Southern China to $100 \%$ (95\% CI 100 to $100 \%$ ) in Hubei, Central China. Overall, the true prevalence of seropositivity was significantly greater in Central China compared with Southern China and significantly greater in Central China compared with Northern China (Table 1).

The prevalence of seropositivity varied by stock class, with higher prevalence estimates in older animals. The true prevalence of $L$. intracellularis seropositivity was significantly greater in boars, sows and fattening pigs compared with pre-weaning piglets and weaners (Table 2). The true prevalence in pre-weaning piglets did not differ significantly from that of weaned piglets. 
Table 1 Antibodies to $L$. intracellularis in commercial pigs by province and region

\begin{tabular}{|c|c|c|c|c|}
\hline \multirow[t]{2}{*}{ Province } & \multicolumn{2}{|c|}{ Number of pigs ${ }^{\#}$} & \multicolumn{2}{|c|}{ Prevalence $(95 \% \mathrm{Cl})^{\dagger}$} \\
\hline & Positive & Tested & Apparent & True \\
\hline \multicolumn{5}{|l|}{ Northern China: } \\
\hline Beijing & 88 & 145 & 61 (41 to 80 ) & 83 (68 to 98 ) \\
\hline Hebei & 87 & 146 & 60 (40 to 79$)$ & 81 (65 to 96) \\
\hline Tianjin & 75 & 146 & 51 (32 to 71) & 68 (50 to 87) \\
\hline Sub-total & 250 & 437 & 57 (46 to 68 ) & $77(68 \text { to } 87)^{a, b}$ \\
\hline \multicolumn{5}{|l|}{ Central China: } \\
\hline Henan & 118 & 187 & 63 (46 to 80) & 86 (74 to 98 ) \\
\hline Hubei & 137 & 188 & 73 (58 to 88) & 100 (100 to 100 \\
\hline Sub-total & 255 & 375 & 68 (57 to 79) & $94(88 \text { to } 100)^{\mathrm{a}, \mathrm{c}}$ \\
\hline \multicolumn{5}{|l|}{ Southern China } \\
\hline Guangdong & 58 & 124 & 47 (25 to 68$)$ & 61 (40 to 82 ) \\
\hline Guanxi & 43 & 124 & 35 (14 to 55) & 43 (22 to 64$)$ \\
\hline Sub-total & 101 & 248 & 41 (26 to 55$)$ & $52(37 \text { to } 67)^{\mathrm{b}, \mathrm{c}}$ \\
\hline Total & 602 & 1060 & 57 (50 to 64) & 77 (70 to 83) \\
\hline
\end{tabular}

"Number of pigs tested, number of pigs positive to the L. intracellularis ELISA test and apparent and true prevalence of $L$. intracellularis seropositivity, by province and region.

${ }^{\dagger}$ Number of pigs seropositive to $L$. intracellularis per 100 pigs tested. $a-a, b-b, c-c$ True prevalence estimates with the same superscript significantly different at the alpha level of 0.05 .

With respect to breed, the true prevalence of seropositivity was higher in Landrace (98\%, $95 \%$ CI 94 to $100 \%)$ and Large White $(96 \%, 95 \%$ CI 90 to 100\%) pigs compared with the local Meishan (60\%, 95\% CI 44 to $76 \%$ ) and Jinhua (37\%, 95\% CI 22 to 52\%) breeds (Table 3). There were no significant differences in the true prevalence of seropositivity for pigs of Landrace and Large White breeds.

The true prevalence of $L$. intracellularis seropositivity in herds with a recent history of diarrhea was $0 \%$ in

Table 2 Seropositivity to $L$. intracellularis in commercial pigs by stock class

\begin{tabular}{|c|c|c|c|c|}
\hline \multirow[t]{2}{*}{ Age class } & \multicolumn{2}{|c|}{ Number of pigs ${ }^{\#}$} & \multicolumn{2}{|c|}{ Prevalence $(95 \% \mathrm{Cl})^{\dagger}$} \\
\hline & Positive & Tested & Apparent & True \\
\hline Pre-weaning & 47 & 147 & $32(14$ to 50$)$ & $38(19 \text { to } 57)^{a}$ \\
\hline Weaners & 58 & 221 & 26 (12 to 40$)$ & $29(15 \text { to } 44)^{b, c, d}$ \\
\hline Fattening & 166 & 279 & 60 (46 to 73$)$ & $81(69 \text { to } 92)^{a, b, e, f}$ \\
\hline Sows & 186 & 255 & $73(60$ to 86$)$ & $100(100 \text { to } 100)^{a, c, e}$ \\
\hline Boars & 145 & 158 & $92(81$ to 100$)$ & $100(100 \text { to } 100)^{a, d, f}$ \\
\hline Total & 602 & 1060 & 57 (50 to 64) & 77 (66 to 87 ) \\
\hline
\end{tabular}

"Number of pigs tested, number of pigs positive to the L. intracellularis ELISA test and apparent and true prevalence of $L$. intracellularis seropositivity, by stock class.

${ }^{\dagger}$ Number of pigs seropositive to L. intracellularis per 100 pigs tested. $a-a, b-b, c-c, d-d, e-e, f-f$ True prevalence estimates with the same superscript significantly different at the alpha level of 0.05 .
Table 3 Seropositivity to L. intracellularis in commercial pigs by breed

\begin{tabular}{llllll}
\hline Breed $^{\#}$ & \multicolumn{2}{c}{ Number of pigs } & & \multicolumn{2}{c}{ Prevalence $(\mathbf{9 5 \%} \mathrm{Cl})^{\dagger}$} \\
\cline { 2 - 3 } \cline { 5 - 6 } & Positive & Tested & & Apparent & True \\
\hline Landrace & 234 & 332 & & $70(59$ to 82$)$ & $98(94 \text { to } 100)^{\mathrm{a}}$ \\
Large White & 199 & 287 & & $69(56$ to 82$)$ & $96(90 \text { to } 100)^{\mathrm{b}, \mathrm{c}}$ \\
Meishan & 97 & 211 & & $46(30$ to 62$)$ & $60(44 \text { to } 76)^{\mathrm{a}, \mathrm{b}}$ \\
Jinhua & 72 & 230 & & $31(17$ to 46$)$ & $37(22 \text { to } 52)^{\mathrm{c}}$ \\
Total & 602 & 1060 & & $57(50$ to 64$)$ & $77(66$ to 87$)$
\end{tabular}

\#Number of pigs tested, number of pigs positive to the $L$. intracellularis ELISA test and apparent and true prevalence of $L$. intracellularis seropositivity, by breed. ${ }^{\dagger}$ Number of pigs seropositive to $L$. intracellularis per 100 pigs tested. $a-a, b-b, c-c$ True prevalence estimates with the same superscript significantly different at the alpha level of 0.05 .

pre-weaning piglets and weaners compared to $100 \%$ in fattening pigs, sows and boars (Table 4). For the fattening pig, sow and boar stock classes the true prevalence of $L$. intracellularis seropositivity was greater in herds that reported a recent history of diarrhea compared with those that did not report diarrhea (Table 4). The confidence limits for the true prevalence of seropositivity in herds with and without a recent history of diarrhea did not overlap for all stock classes except for boars, indicating an association between $L$. intracellularis seropositivity and the presence of clinical diarrhea.

Table 4 Apparent and true $L$. intracellularis seropositivity in pigs from commercial herds by stock class and diarrhea status of the herd

\begin{tabular}{|c|c|c|c|c|}
\hline \multirow[t]{2}{*}{ Breed } & \multicolumn{2}{|c|}{ Number of pigs } & \multicolumn{2}{|c|}{ Prevalence $(95 \% \mathrm{Cl})^{\dagger}$} \\
\hline & Positive & $\overline{\text { Tested }}$ & Apparent & True \\
\hline \multicolumn{5}{|l|}{ Diarrhea present: } \\
\hline Pre-weaning & 2 & 94 & $2(0$ to 9$)$ & $0(0 \text { to } 0)^{a}$ \\
\hline Weaners & 9 & 129 & 7 (0 to 18$)$ & $0(0 \text { to } 21)^{b}$ \\
\hline Fattening & 124 & 159 & 78 (62 to 94$)$ & $100(100 \text { to } 100)^{c}$ \\
\hline Sows & 130 & 157 & 83 (69 to 97) & $100(100 \text { to } 100)^{d}$ \\
\hline Boars & 116 & 120 & 97 (89 to 100$)$ & 100 (100 to 100$)$ \\
\hline Sub-total & 381 & 659 & 58 (49 to 67 ) & 78 (71 to 86$)$ \\
\hline \multicolumn{5}{|l|}{ Diarrhea absent: } \\
\hline Pre-weaning & 45 & 53 & 85 (61 to 100$)$ & $100(100 \text { to } 100)^{\mathrm{a}}$ \\
\hline Weaners & 49 & 92 & 53 (28 to 78$)$ & $71(48 \text { to } 93)^{b}$ \\
\hline Fattening & 42 & 120 & 35 (14 to 56$)$ & $43(22 \text { to } 65)^{c}$ \\
\hline Sows & 56 & 98 & 57 (33 to 81$)$ & $78(57 \text { to } 98)^{d}$ \\
\hline Boars & 29 & 38 & 76 (42 to 100$)$ & 100 (100 to 100$)$ \\
\hline Sub-total & 221 & 401 & 55 (43 to 67 ) & 74 (64 to 84$)$ \\
\hline Total & 602 & 1060 & 57 (50 to 64) & 77 (66 to 87$)$ \\
\hline
\end{tabular}

${ }^{\dagger}$ Number of pigs seropositive to $L$. intracellularis per 100 pigs tested. $a-b, b-b, c-c, d-d$ True prevalence estimates with the same superscript significantly different at the alpha level of 0.05 . 


\section{Discussion}

In this study, using a blocking ELISA, the overall true prevalence of $L$. intracellularis seropositivity was $77 \%$ (95\% CI 70 to 83\%). A higher rate of seroprevalence was found in fattening pigs, sows and boars compared with pre-weaning piglets and weaners. Breeds exotic to China had a higher seroprevalence compared with domestic pig breeds. This is the first report on the infection of L. intracellularis across China. The above evidence indicates that L. intracellularis infection is widespread among intensive pig farms in China.

In this study, none of the pigs had been immunized with $L$. intracellularis attenuated vaccine, but a high seroprevalence was found in the collected serum samples indicating exposure to the bacterium. The apparent seroprevalence estimates in this study are comparable with those reported in other studies. Apparent seroprevalence was $90 \%$ for sows and $56 \%$ for fattening pigs in a study conducted in the USA [23], while growing pigs and fattening pigs were found to be $45 \%$ and $59 \%$ seropositive, respectively, in a Korean study using an immunofluorescent assay as the detection method [9]. In Australia all herds tested positive for L.intracellularisspecific antibodies and the mean within-herd prevalence of positive samples was $84 \%$ [11]. Variation in the sensitivity and specificity of the diagnostic methods used to detect the presence of L.intracellularis contributes to the different serological prevalence estimates reported in Europe and Asia [17,21].

Pigs reared in herds in Central China had true prevalence estimates that were higher compared with pigs reared in herds in the North and South of the country. We speculate that the reason for this finding is that higher seroprevalence rates are associated with intensive pig production units which are common in Central China. The apparent prevalence of $L$. intracellularis seropositivity was similar for sows and boars and markedly higher than that reported for similar stock classes in other countries. Adult sows with active infection are likely to transmit infection to suckling piglets and infected boars transmit pathogens to sows by artificial insemination. Based on a single assessment of a herd's diarrhea status we note that the prevalence of seropositivity in herds with a recent history of clinical diarrhea was similar to that of herds without a recent history of diarrhea which would imply some latency of $L$. intracellularis in sows and boars. The high true prevalence of seropositivity in pre-weaning piglets and boars free of diarrhea does not exclude the possibility that diarrhea was caused by other pathogens. Pig diarrhea is caused by a number of pathogens and mixed infections, including E. coli, Brachyspira hyodysenteriae, Lawsonia intracellularis, and Salmonella spp. [24].

The high true prevalence of $L$. intracellularis seropositivity identified in this study can be explained by a number of factors. First, inappropriate use of in-feed antibiotics contributes to L. intracellularis infection. In some situations there is a risk of animals developing a resistance to antibiotics such as tetracyclines, sulfonamides, fluroquinalones and zinc bacitracin that are routinely used in feed formulations [25]. A recent study based on data collected from pig herds in Southern China reported that antibiotic excretion rates for sows was $48 \mathrm{mg} /$ day, weaning piglets $19 \mathrm{mg} /$ day, growing pigs $7 \mathrm{mg} /$ day and finishing pigs $1.5 \mathrm{mg} /$ day [26] indicating that the usage of antibiotics and therefore excretion masses are correlated with stock class. High $L$. intracellularis seropositivity is known to be associated with withdrawal of in-feed medication in fattening pig diets. In this study, the true prevalence of $L$. intracellularis seropositivity for fattening pigs (81\%, 95\% CI 69 to 92\%) was significantly greater compared with pre-weaning piglets (38\%, 95\% CI 19 to 57\%) and weaners $(29 \%, 95 \%$ CI 15 to $44 \%)$. To some extent, a low true prevalence of $L$. intracellularis seropositivity reflects the presence of long-term treatment with antibiotics [9].

A second explanation for the high true prevalence of L. intracellularis seropositivity is that the presence of infection in breeding herds may arise from the introduction of breeding pigs into a herd without application of appropriate quarantine measures. Every year, thousands of European and American breeding pigs are purchased and imported into China by commercial pig farmers. In most situations the risk awareness of the introducing $L$. intracellularis infection is underestimated and clinical cases of $L$. intracellularis infection are often misdiagnosed by local veterinary authorities as well as the pig purchaser. The apparent prevalence of $L$. intracellularis infection in exotic breeding pigs ranged from $26 \%$ to $74 \%$ in previous reports $[6,27]$. One survey indicated that $56 \%$ of seropositive pigs in Korea originated from pigs born outside of Korea [9]. Recent reports note that L. intracellularis is spread between herds mainly through the purchase of infected pigs and replacement stock [27,28]. The importance of transmission by non-porcine vectors is unknown. Following experimental inoculation, histological lesions develop in laboratory mice, rats and hamsters, but not in sparrows or chickens [29].

In addition to the above, transmission from boars is likely to play a role in the spread of $L$. intracellularis both within and between herds. In this study, the true prevalence in boars could have contributed to infection rates in sows bred by artificial insemination. In the herds that took part in this study, sows were mated using boar semen without antibiotic treatment. The impact of this practice on between-herd spread of infection needs to be clarified.

\section{Conclusions}

We conclude that the prevalence of $L$. intracellularis seropositivity is relatively high in Chinese pigs. The 
highest true prevalence was observed in sows and boars, suggesting that within a herd these stock classes are a reservoir for infection. The prevalence of $L$. intracellularis seropositivity in domestic pigs was significantly less than that in imported breeds. A higher seroprevalence was found in pigs in herds in Central and Northern China, which may correspond to the greater use of the intensive production systems in these areas.

\section{Competing interests}

This work was supported in part by NSFC grant 31172305 and Ministry of Science and Technology (MoST) grant 2012AA101302-4 (Cheng He).

The authors declare that they have no competing interests.

\section{Authors' contributions}

$Z W$, YL and QP contributed to the study design, evaluated the data, and drafted the manuscript. DT was responsible for the coordination of the work and collected blood samples. PH contributed to the revision and $\mathrm{CH}$ contributed to the study design, obtained the funding, and corrected manuscript. All authors have read and approved the final manuscript.

\section{Acknowledgements}

We greatly appreciate the donation of two test kits from Prof. Gregers Jungersen of Technical University of Denmark (DTU) in the preliminary study. Also, we gratefully acknowledge the correcting submission by Dr. Gordon Leitch and the statistical analysis by Dr. Fengxia Yan at Morehouse School of Medicine, Atlanta, USA.

\section{Author details}

${ }^{1}$ Key Lab of Animal Epidemiology and Zoonosis of Ministry of Agriculture, College of Veterinary Medicine, China Agricultural University, Beijing 100193, China. ${ }^{2}$ Innate Immunology Group, National Veterinary Institute, Technical University of Denmark, 1870 Frederiksberg, Denmark.

Received: 10 July 2013 Accepted: 21 April 2014

Published: 28 April 2014

\section{References}

1. Lawson GH, Gebhart CJ: Proliferative enteropathy. J Comp Pathol 2000, 122:77-100

2. McOrist $\mathrm{S}$, Gebhart CJ: Porcine proliferative enteropathies. In Diseases of Swine. 9th edition. Edited by Straw BE. lowa: Blackwell Pub; 2006:1153.

3. Yang HC: Lawsonia. In Veterinary Microbiology, Volume 12. 4th edition. Edited by Lu CP. Beijing: China Agriculture Publishing; 2007:175-176.

4. Guedes RM, Gebhart CJ, Armbruster GA, Roggow BD: Serologic follow-up of a repopulated swine herd after an outbreak of proliferative hemorrhagic enteropathy. Can J Vet Res 2002, 66:258-263.

5. Biester HE, Schwarte LH: Intestinal Adenoma in Swine. Am J Pathol 1931, 7:175-185.

6. Stege $H$, Jensen TK, Møller K, Baekbo P, Jorsal SE: Prevalence of intestinal pathogens in Danish finishing pig herds. Prev Vet Med 2000, 46:279-292.

7. Jacobson M, Gerth Löfstedt M, Holmgren N, Lundeheim N, Fellström C: The prevalence of Brachyspira spp. and Lawsonia intracellularis in Swedish piglet producing herds and wild boar population. J Vet Med B Infect Dis Vet Public Health 2005, 52:386-391.

8. Suh DK, Song JC: Prevalence of Lawsonia intracellularis, Brachyspira hyodysenteriae and Salmonella in swine herds. J Vet Sci 2005, 6:289-293.

9. Lee SW, Kim TJ, Park SY, Song CS, Chang HK, Yeh JK, Park HI, Lee JB: Prevalence of porcine proliferative enteropathy and its control with tylosin in Korea. J Vet Sci 2001, 2:209-212

10. Armbruster GA, Deen J, Gebhart CJ, Pelger GA, Keffaber KK, Parks CW: Review of Lawsonia intracellularis seroprevalence screening in the United States. In Proceedings of the 38th Annual Meet Amer Assoc Swine Vet, 3-6 March 2007: Florida. 2007:231-233.

11. Holyoake PK, Emery D, Gonsalves J, Donahoo M, Collins A: Prevalence of antibodies to Lawsonia intracellularis in pig herds in Australia. Aust Vet $J$ 2010, 88:186-188.

12. Cutler R, Gardner I: A blueprint for pig health research. Canberra, Australia: Australian Pig Research Council Publication; 1989.
13. McOrist S, Smith SH, Green LE: Estimate of direct financial losses due to porcine proliferative enteropathy. Vet record 1997, 140:579-581.

14. Chinese Ministry of Agriculture: Poultry Avian Influenza Vaccination in China. Official Veterinary Bulletin 2006, 10:35-39.

15. Xiao AH, Xie LH, Liao CQ, Lan JL, Li ML, Hou SY, Lu SY, Huang WJ: Analysis of antigenicity in four antigenic candidate genes of Lawsonia intracellularis GXNN strain. Guanxi Agricultural Sciences 2010, 41:62-65. Chinese with English abstract). [www.cnki.net/kcms/detail/detail.aspx?queryID]

16. Otte M, Gumm I: Intra-cluster correlation coefficients of 20 infections calculated from the results of cluster-sample surveys. Prevent Veter Med 1997, 31:147-150.

17. Jacobson M, Wallgren $P$, Nordengrahn A, Merza M, Emanuelson U: Evaluation of a blocking ELISA for the detection of antibodies against Lawsonia intracellularis in pig sera. Acta Vet Scand 2011, 53:23-29.

18. Rogan W, Gladen B: Estimating prevalence from results of a screening test. Am J Epidemiol 1978, 107:71-76.

19. Brown LD, Cat TT, DasGupta A: Interval Estimation for a proportion. Stat Sci 2001, 16:101-133.

20. Reiczigel J, Földi J, Ozsvári L: Exact confidence limits for prevalence of a disease with an imperfect diagnostic test. Epidemiology and Infection 2010, 138:1674-1678.

21. Ventura SJ, Martin JA, Curtin SC, Mathews TJ, Park MM: Births: final data for 1999. National Vital Statistics Reports 2000, $48: 96$

22. EpiTools epidemiological calculators. http://epitools.ausvet.com.au/ content.php?page=home.

23. Corzo CA, Friendship RM, Dewey CE, Blackwell T: Seroprevalence of Lawsonia intracellularis in Ontario swine herds. J Swine Health Prod 2005, 13:314-317.

24. Wendt $M$, Johann RS, Verspohl J: Epidemiological investigations on Lawsonia intracellularis infection in pig herds. Tierarztl Prax 2006, 34:230-9.

25. Li YX, Zhang XL, Li W, Lu XF, Liu B, Wang J: The residues and environmental risks of the multiple veterinary antibiotics in animal faeces. Environ Monit Assess 2013, 185:2211-20.

26. Zhou LJ, Ying GG, Zhang RQ, Liu S, Lai HJ, Chen ZF, Yang B, Zhao JL: Use patterns, excretion masses and contamination profiles of antibiotics in a typical swine farm, south China. Environ Sci Process Impacts 2013, 15:802-13.

27. Chouet S, Prieto C, Mieli L, Veenhuizen MF, McOrist S: Patterns of exposure to Lawsonia intracellularis infection on European pig farms. Vet Rec 2003, 152:14-17.

28. Jacobson M, Aspan A, Nordengrahn A, Lindberg M, Wallgren P: Monitoring of Lawsonia intracellularis in breeding herd gilts. Vet Microbiol 2010, 142:317-22.

29. Collins AM, Love RJ, Jasni S, McOrist S: Attempted infection of mice, rats and chickens by porcine strains of Lawsonia intracellularis. Aust Vet $J$ 1999, 77:120-2.

doi:10.1186/1746-6148-10-100

Cite this article as: Wu et al: Seroprevalence of Lawsonia intracellularis antibodies in intensive pig farms in China. BMC Veterinary Research 2014 10:100.

\section{Submit your next manuscript to BioMed Central and take full advantage of:}

- Convenient online submission

- Thorough peer review

- No space constraints or color figure charges

- Immediate publication on acceptance

- Inclusion in PubMed, CAS, Scopus and Google Scholar

- Research which is freely available for redistribution 